\title{
$q$-deformed $\mathcal{W}$-algebras and elliptic algebras
}

\author{
J. Avan \\ LPTHE, CNRS-URA 280, Universités Paris VI/VII, France \\ L. Frappat, M. Rossi, P. Sorba \\ Laboratoire d'Annecy-le-Vieux de Physique Théorique LAPTH, CNRS-URA 1436 \\ LAPP, BP 110, F-74941 Annecy-le-Vieux Cedex, France
}

\begin{abstract}
The elliptic algebra $\mathcal{A}_{q, p}\left(\widehat{s l}(N)_{c}\right)$ at the critical level $c=-N$ has an extended center containing trace-like operators $t(z)$. Families of Poisson structures, defining $q$-deformations of the $\mathcal{W}_{N}$ algebra, are constructed. The operators $t(z)$ also close an exchange algebra when $\left(-p^{1 / 2}\right)^{N M}=q^{-c-N}$ for $M \in \mathbb{Z}$. It becomes Abelian when in addition $p=q^{N h}$ where $h$ is a non-zero integer. The Poisson structures obtained in these classical limits contain different $q$-deformed $\mathcal{W}_{N}$ algebras depending on the parity of $h$, characterizing the exchange structures at $p \neq q^{N h}$ as new $\mathcal{W}_{q, p}(s l(N))$ algebras.
\end{abstract}

MSC number: 81R50, 17B37

Talk presented by L. Frappat at the 7th International Colloquium "Quantum Groups and Integrable Systems" (QGIS), Prague, 18-20 June 1998.

math.QA/9807131

July 1998 


\section{Introduction}

The results hereafter presented are based on four papers of the authors [1-3]. They deal with the construction of $q$-deformed Virasoro, and more generally $\mathcal{W}$, algebras in relation with the quantum elliptic algebras $\mathcal{A}_{q, p}\left(\widehat{s l}(N)_{c}\right)$. Indeed what we achieve here is really a $q$-deformation of the Sugawara construction on an abstract level (that is only abstract algebraic relations in $\mathcal{A}_{q, p}\left(\widehat{s l}(N)_{c}\right)$ are used).

We first prove the existence of an extended center in $\mathcal{A}_{q, p}\left(\widehat{s l}(N)_{c}\right)$ at $c=-N$ and compute the Poisson structures on this center. These structures are identified as $q$-deformed $V i r_{q}(\operatorname{sl}(N))$ algebras. We then show the existence of closed (quadratic) exchange algebras whenever $\left(-p^{\frac{1}{2}}\right)^{N M}=q^{-c-N}$ for any integer $M \in \mathbb{Z}$. These algebras admit a classical limit (commuting algebras) at $p=q^{N h}$ with $h \in \mathbb{Z} \backslash\{0\}$. The related Poisson structures include for $h$ even the Poisson structures in [ $⿴ 囗 十$ ]. The exchange algebras therefore realize new quantizations of these Poisson structures. When $h$ is odd, by contrast, this classical limit takes a form different from the initial $\operatorname{Vir}_{q}(s l(N))$ structures. This emphasizes the key role of the initial 3-parameter structure $\mathcal{A}_{q, p}\left(\widehat{s l}(N)_{c}\right)$ in allowing for an intermediate quantum $q$-deformed Virasoro algebra. When computing the mode expansion of these structures (both classical and quantum), a "sector-type" labeling is needed due to the singularity behaviour of the structure function viewed as an analytic continuation and therefore exhibiting different formal series expansions corresponding to different convergent series expansions in distinct domains of the complex plane. Finally, we describe the essential features of the extension of our computation to higher spin fields.

\section{The elliptic algebra $\mathcal{A}_{q, p}\left(\widehat{s l}(N)_{c}\right)$}

We start by defining the $R$-matrix of the $\mathbb{Z}_{N}$-vertex model $\left(\mathbb{Z}_{N} \equiv \mathbb{Z} / N \mathbb{Z}\right)$ [5, 6]:

$$
\widetilde{R}(z, q, p)=\frac{z^{2 / N-2}}{\kappa\left(z^{2}\right)} \frac{\vartheta\left[\begin{array}{c}
\frac{1}{2} \\
\frac{1}{2}
\end{array}\right](\zeta, \tau)}{\vartheta\left[\begin{array}{c}
\frac{1}{2} \\
\frac{1}{2}
\end{array}\right](\xi+\zeta, \tau)} \sum_{\left(\alpha_{1}, \alpha_{2}\right)} W_{\left(\alpha_{1}, \alpha_{2}\right)}(\xi, \zeta, \tau) I_{\left(\alpha_{1}, \alpha_{2}\right)} \otimes I_{\left(\alpha_{1}, \alpha_{2}\right)}^{-1},
$$

where $z=e^{i \pi \xi}, q=e^{i \pi \zeta}, p=e^{2 i \pi \tau}$ and $\left(\alpha_{1}, \alpha_{2}\right) \in \mathbb{Z}_{N} \times \mathbb{Z}_{N}$.

$\vartheta$ are the Jacobi theta functions with rational characteristics $\left(\gamma_{1}, \gamma_{2}\right) \in \mathbb{Z} / N \times \mathbb{Z} / N$ :

$$
\vartheta\left[\begin{array}{l}
\gamma_{1} \\
\gamma_{2}
\end{array}\right](\xi, \tau)=\sum_{m \in \mathbb{Z}} \exp \left(i \pi\left(m+\gamma_{1}\right)^{2} \tau+2 i \pi\left(m+\gamma_{1}\right)\left(\xi+\gamma_{2}\right)\right) \text {. }
$$

The normalization factor is given by:

$$
\frac{1}{\kappa\left(z^{2}\right)}=\frac{\left(q^{2 N} z^{-2} ; p, q^{2 N}\right)_{\infty}\left(q^{2} z^{2} ; p, q^{2 N}\right)_{\infty}\left(p z^{-2} ; p, q^{2 N}\right)_{\infty}\left(p q^{2 N-2} z^{2} ; p, q^{2 N}\right)_{\infty}}{\left(q^{2 N} z^{2} ; p, q^{2 N}\right)_{\infty}\left(q^{2} z^{-2} ; p, q^{2 N}\right)_{\infty}\left(p z^{2} ; p, q^{2 N}\right)_{\infty}\left(p q^{2 N-2} z^{-2} ; p, q^{2 N}\right)_{\infty}}
$$

The functions $W_{\left(\alpha_{1}, \alpha_{2}\right)}$ are defined as follows:

$$
W_{\left(\alpha_{1}, \alpha_{2}\right)}(\xi, \zeta, \tau)=\frac{1}{N} \frac{\vartheta\left[\begin{array}{c}
\frac{1}{2}+\alpha_{1} / N \\
\frac{1}{2}+\alpha_{2} / N
\end{array}\right](\xi+\zeta / N, \tau)}{\vartheta\left[\begin{array}{c}
\frac{1}{2}+\alpha_{1} / N \\
\frac{1}{2}+\alpha_{2} / N
\end{array}\right](\zeta / N, \tau)}
$$


and the matrices $I_{\left(\alpha_{1}, \alpha_{2}\right)}$ by $I_{\left(\alpha_{1}, \alpha_{2}\right)}=g^{\alpha_{2}} h^{\alpha_{1}}$ where $g_{i j}=\omega^{i} \delta_{i j}, h_{i j}=\delta_{i+1, j}$ are $N \times N$ matrices (the addition of indices being understood modulo $N)$ and $\omega=e^{2 i \pi / N}$.

The $R$-matrix $\widetilde{R}$ is $\mathbb{Z}_{N}$-symmetric:

$$
\widetilde{R}_{c+s, d+s}^{a+s+s}=\widetilde{R}_{c, d}^{a, b} \quad a, b, c, d, s \in \mathbb{Z}_{N}
$$

We introduce the "gauge-transformed" matrix:

$$
R(z, q, p)=\left(g^{\frac{1}{2}} \otimes g^{\frac{1}{2}}\right) \widetilde{R}(z, q, p)\left(g^{-\frac{1}{2}} \otimes g^{-\frac{1}{2}}\right) .
$$

It satisfies the following properties:

- Yang-Baxter equation: $R_{12}(z) R_{13}(w) R_{23}(w / z)=R_{23}(w / z) R_{13}(w) R_{12}(z)$,

- unitarity: $R_{12}(z) R_{21}\left(z^{-1}\right)=1$,

- crossing symmetry: $R_{12}(z)^{t_{2}} R_{21}\left(q^{-N} z^{-1}\right)^{t_{2}}=1$,

- antisymmetry: $R_{12}(-z)=\omega\left(g^{-1} \otimes \mathbb{I}\right) R_{12}(z)(g \otimes \mathbb{I})$,

- quasi-periodicity: $\widehat{R}_{12}\left(-p^{\frac{1}{2}} z\right)=\left(g^{\frac{1}{2}} h g^{\frac{1}{2}} \otimes \mathbb{I}\right)^{-1}\left(\widehat{R}_{21}\left(z^{-1}\right)\right)^{-1}\left(g^{\frac{1}{2}} h g^{\frac{1}{2}} \otimes \mathbb{I}\right)$,

where $\widehat{R}_{12}(x)=\tau_{N}\left(q^{1 / 2} x^{-1}\right) R_{12}(x)$ and $\tau_{N}(z)=z^{\frac{2}{N}-2} \frac{\Theta_{q^{2 N}}\left(q z^{2}\right)}{\Theta_{q^{2 N}\left(q z^{-2}\right)}}$. The function $\tau_{N}(z)$ is periodic with period $q^{N}: \tau_{N}\left(q^{N} z\right)=\tau_{N}(z)$ and satisfies $\tau_{N}\left(z^{-1}\right)=\tau_{N}(z)^{-1}$.

We now define the elliptic quantum algebra $\mathcal{A}_{q, p}\left(\widehat{s l}(N)_{c}\right)$ [7, 8]. It is an algebra of operators $L_{i j}(z) \equiv \sum_{n \in \mathbb{Z}} L_{i j}(n) z^{n}$ where $i, j \in \mathbb{Z}_{N}$ :

$$
L(z)=\left(\begin{array}{ccc}
L_{11}(z) & \cdots & L_{1 N}(z) \\
\vdots & & \vdots \\
L_{N 1}(z) & \cdots & L_{N N}(z)
\end{array}\right) .
$$

The $q$-determinant is given by $(\varepsilon(\sigma)$ being the signature of the permutation $\sigma)$ :

$$
q-\operatorname{det} L(z) \equiv \sum_{\sigma \in \mathfrak{S}_{N}} \varepsilon(\sigma) \prod_{i=1}^{N} L_{i, \sigma(i)}\left(z q^{i-N-1}\right) \text {. }
$$

$\mathcal{A}_{q, p}\left(\widehat{g l}(N)_{c}\right)$ is defined by imposing the following constraints on $L(z)$ :

$$
\widehat{R}_{12}(z / w) L_{1}(z) L_{2}(w)=L_{2}(w) L_{1}(z) \widehat{R}_{12}^{*}(z / w),
$$

where $L_{1}(z) \equiv L(z) \otimes \mathbb{I}, L_{2}(z) \equiv \mathbb{I} \otimes L(z)$ and $\widehat{R}_{12}^{*}(z, q, p) \equiv \widehat{R}_{12}\left(z, q, p^{*}=p q^{-2 c}\right)$.

The $q$-determinant is in the center of $\mathcal{A}_{q, p}\left(\widehat{g l}(N)_{c}\right)$ and one sets

$$
\mathcal{A}_{q, p}\left(\widehat{s l}(N)_{c}\right)=\mathcal{A}_{q, p}\left(\widehat{g l}(N)_{c}\right) /\left\langle q-\operatorname{det} L-q^{\frac{c}{2}}\right\rangle
$$

\section{The center of $\mathcal{A}_{q, p}\left(\widehat{s l}(N)_{c}\right)$}

We set $L^{+}(z) \equiv L\left(q^{c / 2} z\right)$ and $L^{-}(z) \equiv g^{\frac{1}{2}} h g^{\frac{1}{2}} L\left(-p^{\frac{1}{2}} z\right)\left(g^{\frac{1}{2}} h g^{\frac{1}{2}}\right)^{-1}$. Let us define the following operator in $\mathcal{A}_{q, p}\left(\widehat{s l}(N)_{c}\right)$ :

$$
t(z) \equiv \operatorname{Tr}(\mathcal{L}(z)) \equiv \operatorname{Tr}\left(L^{+}\left(q^{c / 2} z\right) L^{-}(z)^{-1}\right)
$$


From the defining relations of the elliptic algebra and the properties of the matrix $\widehat{R}$ (especially the quasi-periodicity property), one gets the following theorems:

Theorem 1: At the critical level $c=-N$, the operators generated by $t(z)$ lie in the center of the algebra $\mathcal{A}_{q, p}\left(\widehat{s l}(N)_{c}\right)$ :

$$
\left[t(z), L^{+}(w)\right]=\left[t(z), L^{-}(w)\right]=0 .
$$

Theorem 2: At the critical level $c=-N$, the operators generated by $t(z)$ close an Abelian algebra:

$$
[t(z), t(w)]=0
$$

This allows us to define a Poisson structure on the center of $\mathcal{A}_{q, p}\left(\widehat{s l}(N)_{c}\right)$ at $c=-N$. When $c \neq-N$, one has:

$$
\begin{aligned}
t(z) t(w) & =\mathcal{Y}(w / z)_{j_{1} j_{2}}^{i_{1} i_{2}} \mathcal{L}(w)_{i_{2}}^{j_{2}} \mathcal{L}(z)_{i_{1}}^{j_{1}} \\
& =t(w) t(z)+\left.(c+N)\left(\frac{d \mathcal{Y}}{d c}\left(\frac{w}{z}\right)\right)_{j_{1} j_{2}}^{i_{1} i_{2}} \mathcal{L}(w)_{i_{2}}^{j_{2}} \mathcal{L}(z)_{i_{1}}^{j_{1}}\right|_{c r}+\cdots
\end{aligned}
$$

The Poisson structure is then defined by:

$$
\{t(z), t(w)\}=\left.\left(\frac{d \mathcal{Y}}{d c}\left(\frac{w}{z}\right)\right)_{j_{1} j_{2}}^{i_{1} i_{2}} \mathcal{L}(w)_{i_{2}}^{j_{2}} \mathcal{L}(z)_{i_{1}}^{j_{1}}\right|_{c r} .
$$

Decomposing $\mathcal{Y}(x)$ as $\mathcal{Y}(x)=T(x) \mathcal{M}(x)$ where

$$
T(x)=\frac{\tau_{N}\left(q^{\frac{1}{2}} x^{-1}\right) \tau_{N}\left(q^{\frac{1}{2}-c} x\right)}{\tau_{N}\left(q^{\frac{1}{2}} x\right) \tau_{N}\left(q^{\frac{1}{2}-c} x^{-1}\right)}
$$

and

$$
\mathcal{M}(x)=\left(\left(R_{21}(x) R_{21}\left(q^{c+N} x\right)^{-1} R_{12}\left(x^{-1}\right)^{-1}\right)^{t_{2}} R_{12}\left(q^{c} x^{-1}\right)^{t_{2}}\right)^{t_{2}}
$$

one gets

$$
\left.\frac{d \mathcal{Y}}{d c}(x)\right|_{c r}=\left.\frac{d T}{d c}(x)\right|_{c r} \mathbb{I}_{2} \otimes \mathbb{I}_{2}+\left.\frac{d \mathcal{M}}{d c}(x)\right|_{c r},
$$

since $T(x)_{c r}=1$ and $\mathcal{M}(x)_{c r}=\mathbb{I}_{2} \otimes \mathbb{I}_{2}$ at the critical level $c=-N$.

The properties of the matrix $R_{12}$ imply the vanishing of the derivative of $\mathcal{M}$ with respect to $c$ at the critical level and it follows that:

$$
\{t(z), t(w)\}=\left.\frac{d T}{d c}\left(\frac{w}{z}\right)\right|_{c r} t(z) t(w) \equiv f(w / z) t(z) t(w)
$$

where

$$
\begin{aligned}
f(x)=-2 \ln q & {\left[\sum_{l \geq 0}\left(\frac{2 x^{2} q^{2 N l}}{1-x^{2} q^{2 N l}}-\frac{x^{2} q^{2 N l+2}}{1-x^{2} q^{2 N l+2}}-\frac{x^{2} q^{2 N l-2}}{1-x^{2} q^{2 N l-2}}\right)\right.} \\
& \left.-\frac{x^{2}}{1-x^{2}}+\frac{1}{2} \frac{x^{2} q^{2}}{1-x^{2} q^{2}}+\frac{1}{2} \frac{x^{2} q^{-2}}{1-x^{2} q^{-2}}-\left(x \leftrightarrow x^{-1}\right)\right] .
\end{aligned}
$$

The Poisson structure on the center of $\mathcal{A}_{q, p}\left(\widehat{s l}(N)_{c}\right)$ at $c=-N$ is not $p$ depending. 
We now define the Poisson structure for modes of the generating function $f(x)$. The modes of $t(z)$ are defined in the sense of formal series expansions: $t_{m}=\oint_{C} \frac{d z}{2 i \pi z} z^{-m} t(z)$, where $C$ is a contour encircling the origin.

However, the function $f(x)$ has poles for $x= \pm q^{ \pm N l}$ and $x= \pm q^{ \pm N l \pm 1}$ with $l \geq 0$, therefore

$$
\oint_{C_{1}} \frac{d z}{2 \pi i z} \oint_{C_{2}} \frac{d w}{2 \pi i w} \neq \oint_{C_{2}} \frac{d z}{2 \pi i z} \oint_{C_{1}} \frac{d w}{2 \pi i w} \text {. }
$$

The relative position of the contours $C_{1}$ and $C_{2}$ must be specified in order to have an unambiguous result for the Poisson bracket. Moreover, the antisymmetry of the Poisson bracket is only guaranteed at the mode level by an explicit symmetrization with respect to the position of the contours $C_{1}$ and $C_{2}$. The mode Poisson bracket is thus defined as:

$$
\begin{array}{r}
\left\{t_{n}, t_{m}\right\}=\frac{1}{2}\left(\oint_{C_{1}} \frac{d z}{2 \pi i z} \oint_{C_{2}} \frac{d w}{2 \pi i w}+\oint_{C_{2}} \frac{d z}{2 \pi i z} \oint_{C_{1}} \frac{d w}{2 \pi i w}\right) \\
z^{-n} w^{-m} f(w / z) t(z) t(w)
\end{array}
$$

where $C_{1}$ and $C_{2}$ are circles of radii $R_{1}$ and $R_{2}$ and one chooses $R_{1}>R_{2}$.

The Poisson bracket depends on the relative positions of the contours $C_{1}$ and $C_{2}$, circles of radii $R_{1}$ and $R_{2}$. Thus we introduce the notion of sectors: the sector $(k)$ is defined by $R_{1} / R_{2} \in$ ]$|q|^{-P(k)},|q|^{-P(k+1)}\left[\right.$ where $\{P(k)\}$ is the ordered set of powers of $q^{-1}$ where the poles of $f$ are located: $\{P(k)\}=\{0,1 ; N-1, N, N+1 ; \ldots ; N l-1, N l, N l+1 ; \ldots\}$.

In the case of $s l(N)$, for $\left.R_{1} / R_{2} \in\right] 1,|q|^{-1}$ [ (first sector), one gets the following formula:

$$
\left\{t_{n}, t_{m}\right\}_{k=0}=-2\left(q-q^{-1}\right) \ln q \sum_{r \in \mathbb{Z}} \frac{[(N-1) r]_{q}[r]_{q}}{[N r]_{q}} t_{n-2 r} t_{m+2 r}
$$

where $[r]_{q}$ are $q$-numbers: $[r]_{q} \equiv \frac{q^{r}-q^{-r}}{q-q^{-1}}$.

In the case of $s l(2)$, for $\left.R_{1} / R_{2} \in\right]|q|^{ \pm k},|q|^{ \pm(k+1)}$ [, it is possible to get a nice compact formula for any sector:

$$
\begin{aligned}
\left\{t_{n}, t_{m}\right\}_{k}= & (-1)^{k+1} 2 \ln q \oint_{C_{1}} \frac{d z}{2 \pi i z} \oint_{C_{2}} \frac{d w}{2 \pi i w} \\
& \sum_{s \in \mathbb{Z}} \frac{q^{(2 k+1) s}-q^{-(2 k+1) s}}{q^{s}+q^{-s}}\left(\frac{z}{w}\right)^{2 s} z^{-n} w^{-m} t(z) t(w) .
\end{aligned}
$$

It follows that one obtains in this way a family of Poisson structures indexed by $k \in \mathbb{Z}$, the sector $k=0$ corresponding to the Poisson bracket in 国.

To realize deformed $\mathcal{W}_{N}$ Poisson structures, we need to introduce generating functions for the higher spin objects. Having at our disposal only one commuting generating function $t(z)$, we are led to define shifted products, although with the same generator. We define therefore the higher spin objects for $1 \leq i \leq N-1$ as:

$$
s_{i}(z)=\prod_{u=-(i-1) / 2}^{(i-1) / 2} t\left(q^{u} z\right)
$$


The Poisson algebra reads as:

$$
\left\{s_{i}(z), s_{j}(w)\right\}=\sum_{u=-(i-1) / 2}^{(i-1) / 2} \sum_{v=-(j-1) / 2}^{(j-1) / 2} f\left(q^{v-u} \frac{w}{z}\right) s_{i}(z) s_{j}(w) .
$$

As before, one can introduce the notion of sectors for the Poisson brackets of the mode expansions, now labelled by $k(i, j)$ [3].

For the sector $k=0$, one gets:

$$
\begin{aligned}
\left\{s_{i}(n), s_{j}(m)\right\}= & -\sum_{r \in \mathbb{Z}} \frac{[(N-\max (i, j)) r]_{q}[\min (i, j) r]_{q}}{[N r]_{q}} \\
& \times 2\left(q-q^{-1}\right) \ln q s_{i}(n-2 r) s_{j}(m+2 r) .
\end{aligned}
$$

Any Poisson structure in a given sector $k(i, j)$ can be obtained from $\left(^{*}\right)$ by adding to the $r$-dependent structure coefficient contributions from the relevant singularities of the structure function, i.e. power series expansions of terms $\delta\left( \pm q^{ \pm P_{i j}(s)} w / z\right) s_{i}(z) s_{j}(w)$ for $1 \leq s \leq k(i, j)$.

\section{$4 \mathcal{W}_{q, p}(\operatorname{sl}(N))$ exchange algebras}

\section{1 $q$-Virasoro exchange algebras}

While for $c=-N, t(z)$ and $L(w)$ commute, for $\left(-p^{\frac{1}{2}}\right)^{N M}=q^{-c-N}(M \in \mathbb{Z}), t(z)$ and $L(w)$ realize an exchange algebra:

$$
t(z) L(w)=F(M, w / z) L(w) t(z)
$$

where

$$
F(M, x)=\left\{\begin{array}{cl}
q^{2 M(N-1)} \prod_{k=0}^{N M-1} \frac{\Theta_{q^{2 N}}\left(x^{-2} p^{-k}\right) \Theta_{q^{2 N}}\left(x^{2} p^{k}\right)}{\Theta_{q^{2 N}}\left(x^{-2} q^{2} p^{-k}\right) \Theta_{q^{2 N}}\left(x^{2} q^{2} p^{k}\right)} & \text { for } M>0, \\
q^{-2|M|(N-1)} \prod_{k=1}^{N|M|} \frac{\Theta_{q^{2 N}}\left(x^{-2} q^{2} p^{k}\right) \Theta_{q^{2 N}}\left(x^{2} q^{2} p^{-k}\right)}{\Theta_{q^{2 N}}\left(x^{-2} p^{k}\right) \Theta_{q^{2 N}}\left(x^{2} p^{-k}\right)} & \text { for } M<0 .
\end{array}\right.
$$

It follows that one gets the following theorem:

Theorem 3: On the 2d-surface $\Sigma_{N, M}=\left\{(p, q) \mid\left(-p^{\frac{1}{2}}\right)^{N M}=q^{-c-N}\right\}$ of the 3d-space of parameters $q, p, c$, the operators generated by $t(z)$ close a quadratic algebra:

$$
t(z) t(w)=\mathcal{Y}_{N, p, q, M}\left(\frac{w}{z}\right) t(w) t(z)
$$

where

$$
\mathcal{Y}_{N, p, q, M}(x)= \begin{cases}\prod_{k=1}^{N M} \frac{\Theta_{q^{2 N}}^{2}\left(x^{2} p^{-k}\right) \Theta_{q^{2 N}}\left(x^{2} q^{2} p^{k}\right) \Theta_{q^{2 N}}\left(x^{2} q^{-2} p^{k}\right)}{\Theta_{q^{2 N}}^{2}\left(x^{2} p^{k}\right) \Theta_{q^{2 N}}\left(x^{2} q^{2} p^{-k}\right) \Theta_{q^{2 N}}\left(x^{2} q^{-2} p^{-k}\right)} & \text { for } M>0, \\ \prod_{k=1}^{N|M|-1} \frac{\Theta_{q^{2 N}}^{2}\left(x^{2} p^{-k}\right) \Theta_{q^{2 N}}\left(x^{2} q^{2} p^{k}\right) \Theta_{q^{2 N}}\left(x^{2} q^{-2} p^{k}\right)}{\Theta_{q^{2 N}}^{2}\left(x^{2} p^{k}\right) \Theta_{q^{2 N}}\left(x^{2} q^{2} p^{-k}\right) \Theta_{q^{2 N}}\left(x^{2} q^{-2} p^{-k}\right)} & \text { for } M<0 .\end{cases}
$$


For $N=2$, one has

$$
\begin{aligned}
& \mathcal{Y}_{2, p, q, M}\left(x q^{2}\right)=\mathcal{Y}_{2, p, q, M}(x), \\
& \mathcal{Y}_{2, p, q, M}(x) \mathcal{Y}_{2, p, q, M}(x q)=1
\end{aligned}
$$

One recovers the same periodicity properties satisfied by structure function $\varphi(x)$ of [9] and [10] in the case of $\operatorname{sl}(2)$ (when $M=1$, one makes the change of variable $q^{2} \rightarrow p, p \rightarrow q, x^{2} \rightarrow x$, then $\mathcal{Y}_{2, p, q, 1}(x) \rightarrow \varphi^{2}(x)$ of $\left.[10]\right)$.

For $N>2$, one has

$$
\begin{aligned}
& \mathcal{Y}_{N, p, q, M}\left(x q^{N}\right)=\mathcal{Y}_{N, p, q, M}(x) \\
& \mathcal{Y}_{N, p, q, M}(x) \mathcal{Y}_{N, p, q, M}(x q) \ldots \mathcal{Y}_{N, p, q, M}\left(x q^{N-1}\right)=1
\end{aligned}
$$

\section{2 $\mathcal{W}_{q, p}(\operatorname{sl}(N))$ exchange algebras}

The generalization to higher spin generators in the quantum case follows that of the classical case, although with a required notion of ordering between individual $t(z)$-generators:

$$
s_{i}(z)=\prod_{\frac{i-1}{2} \geq u \geq-\frac{i-1}{2}}^{\curvearrowleft} t\left(q^{u} z\right) .
$$

The exchange algebra takes now the form:

$$
s_{i}(z) s_{j}(w)=\prod_{u=-\frac{i-1}{2}}^{\frac{i-1}{2}} \prod_{v=-\frac{j-1}{2}}^{\frac{j-1}{2}} \mathcal{Y}_{N, p, q, M}\left(q^{v-u} \frac{w}{z}\right) s_{j}(w) s_{i}(z) .
$$

Furthermore one can verify that $s_{N}(z)$ commutes with all other generators; hence we are justified in restricting $i$ to $1, \ldots, N-1$.

\subsection{Classical limit}

Theorem 4: On the surface $\Sigma_{N, M}$, when $p=q^{N h}$ with $h \in \mathbb{Z} \backslash\{0\}$ :

$$
\mathcal{Y}_{N, p, q, M}=1 \text {. }
$$

Hence $t(z)$ realizes an Abelian subalgebra in $\mathcal{A}_{q, p}\left(\widehat{s l}(N)_{c}\right)$ (though it is not in the center of $\mathcal{A}_{q, p}\left(\widehat{s l}(N)_{c}\right)$ in general).

The result of the previous theorem allows us to define Poisson structures on the corresponding Abelian algebras generated by $t(z)$. They are obtained as limits of the exchange algebra of Theorem 3 when $p=q^{N h}$ with $h \in \mathbb{Z} \backslash\{0\}$. Setting $q^{N h}=p^{1-\beta}$, the $h$-labeled Poisson structure is defined by:

$$
\{t(z), t(w)\}^{(h)} \equiv \lim _{\beta \rightarrow 0} \frac{1}{\beta}(t(z) t(w)-t(w) t(z)) .
$$

For $h$ even, $\{t(z), t(w)\}^{(h)}$ coincides with the Poisson structure of the critical case $c=-N$, while for $h$ odd, $\{t(z), t(w)\}^{(h)}$ gives new Poisson structures (for $\operatorname{sl}(N)$ when $N>2$ ). Explicitly, one has the following expression:

$$
\{t(z), t(w)\}^{(h)}=f_{h}(w / z) t(z) t(w)
$$


with

$$
\begin{aligned}
f_{h}(x)= & \mathcal{N}_{\text {odd }}\left[\sum _ { \ell \geq 0 } E ( \frac { N M } { 2 } ) ( E ( \frac { N M } { 2 } ) + 1 ) \left(\frac{2 x^{2} q^{2 N \ell}}{1-x^{2} q^{2 N \ell}}-\frac{x^{2} q^{2 N \ell+2}}{1-x^{2} q^{2 N \ell+2}}\right.\right. \\
& \left.-\frac{x^{2} q^{2 N \ell-2}}{1-x^{2} q^{2 N \ell-2}}\right)+E\left(\frac{N M+1}{2}\right)^{2}\left(\frac{2 x^{2} q^{2 N \ell+N}}{1-x^{2} q^{2 N \ell+N}}-\frac{x^{2} q^{2 N \ell+N+2}}{1-x^{2} q^{2 N \ell+N+2}}\right. \\
& \left.-\frac{x^{2} q^{2 N \ell+N-2}}{1-x^{2} q^{2 N \ell+N-2}}\right)-\frac{1}{2} E\left(\frac{N M}{2}\right)\left(E\left(\frac{N M}{2}\right)+1\right)\left(\frac{2 x^{2}}{1-x^{2}}-\frac{x^{2} q^{2}}{1-x^{2} q^{2}}\right. \\
& \left.\left.-\frac{x^{2} q^{-2}}{1-x^{2} q^{-2}}\right)-\left(x \leftrightarrow x^{-1}\right)\right] \\
= & \mathcal{N}_{\text {even }}\left[\sum_{\ell \geq 0}\left(\frac{2 x^{2} q^{2 N \ell}}{1-x^{2} q^{2 N \ell}}-\frac{x^{2} q^{2 N \ell+2}}{1-x^{2} q^{2 N \ell+2}}-\frac{x^{2} q^{2 N \ell-2}}{1-x^{2} q^{2 N \ell-2}}\right)\right. \\
& \left.-\frac{1}{2}\left(\frac{2 x^{2}}{1-x^{2}}-\frac{x^{2} q^{2}}{1-x^{2} q^{2}}-\frac{x^{2} q^{-2}}{1-x^{2} q^{-2}}\right)-\left(x \leftrightarrow x^{-1}\right)\right] \quad \text { for } h \text { even }
\end{aligned}
$$

where $\mathcal{N}_{\text {odd }}=2 N h \ln q, \mathcal{N}_{\text {even }}=N^{2} M(N M+1) h \ln q$ and the notation $E(n)$ means the integer part of the number $n$.

The mode Poisson brackets in the sector $k=0$ is given by:

$$
\begin{aligned}
&\left\{t_{n}, t_{m}\right\}^{(h)=}-2\left(q-q^{-1}\right) \ln q \sum_{r \in \mathbb{Z}}\left(-E\left(\frac{N M+1}{2}\right)^{2} \frac{[r]_{q}^{2}}{[N r]_{q}}\right. \\
&\left.+E\left(\frac{N M}{2}\right)\left(E\left(\frac{N M}{2}\right)+1\right) \frac{[(N-1) r]_{q}[r]_{q}}{[N r]_{q}}\right) t_{n-2 r} t_{m+2 r} \\
& \text { for } h \text { odd } \\
&=-2\left(q-q^{-1}\right) \ln q \sum_{r \in \mathbb{Z}} \frac{[(N-1) r]_{q}[r]_{q}}{[N r]_{q}} t_{n-2 r} t_{m+2 r} \\
& \text { for } h \text { even }
\end{aligned}
$$

Since the initial non-abelian structure for $t(z)$ is closed, the exchange algebras

$$
t(z) t(w)=\mathcal{Y}_{N, p, q, M}(w / z) t(w) t(z)
$$

are inequivalent quantizations with respect to $p$ of the Poisson bracket which defines $\operatorname{Vir} r_{q}(s l(N))$ : $t(z)$ generates a $\operatorname{Vir}_{q, p}(\operatorname{sl}(N))$ algebra.

The generalization for the higher spin generators $s_{i}(z)$ presents no conceptual difficulty. The following results are obtained:

- for $h$ even, $\left\{s_{i}(z), s_{j}(w)\right\}^{(h)}$ coincides with the Poisson structure of the critical case $c=-N$. The Poisson bracket is again identical to the "core" contribution in [四] (i.e. without lower-spin extensions).

- for $h$ odd, $\left\{s_{i}(z), s_{j}(w)\right\}^{(h)}$ gives rise to two new types of quadratic Poisson structures (that is $q$-deformed classical $\mathcal{W}_{N}$-algebras) whether $i+j \leq N$ or $i+j>N$. 


\section{Conclusion}

Here above is summarized the construction, from elliptic algebras, of the deformed Virasoro and $\mathcal{W}$ algebras in the classical case as well as in the quantum case. We can mention that a study for consistent central extensions, providing in particular general results for the simplest cases of $\operatorname{Vir}_{q}(s l(2))$ has very recently been achieved in ref. [11]. Also, a direct determination of abstract generators for $q$-deformed $\mathcal{W}_{N}$ algebras has been carried out. Such an universal construction of $\mathcal{W}_{q, p}$ algebras is achieved in [12].

An important application of the above developed constructions is the determination of integrable models submitted to such symmetries. It is known that the trigonometric Calogero-Moser model is related to the Virasoro algebra and the Ruijsenaars-Schneider model to the $q$-deformed Virasoro algebra considered in ref. [9]. One expects the existence of generalized Ruijsenaars-Schneider models the symmetries of which would be given by the just described $q$-Virasoro exchange algebras. For such a program, the bosonization of these algebras appears as an essential tool, which is still missing.

\section{References}

[1] Avan J., Frappat L., Rossi M., and Sorba P.: Phys. Lett. A 235 (1997) 323.

[2] Avan J., Frappat L., Rossi M., and Sorba P.: Phys. Lett. A 239 (1998) 27.

[3] Avan J., Frappat L., Rossi M., and Sorba P.: to appear in Commun. Math. Phys., math.QA/9801105.

[4] Frenkel E., Reshetikhin N.: Commun. Math. Phys. 178 (1996) 237.

[5] Baxter R. J.: "Exactly solved models in statistical mechanics", Academic Press, London, 1982.

[6] Belavin A. A.: Nucl. Phys. B 180 (1981) 189.

[7] Foda O., Iohara K., Jimbo M., Kedem R., Miwa T., and Yan H.: Lett. Math. Phys. 32 (1994) 259.

[8] Jimbo M., Konno H., Odake S., and Shiraishi J.: q-alg/9712029.

[9] Shiraishi J., Kubo H., Awata H., Odake S., Lett. Math. Phys. 38 (1996) 33.

[10] Feigin B., Frenkel E., Commun. Math. Phys. 178 (1996) 653.

[11] Avan J., Frappat L., Rossi M., and Sorba P.: submitted to Phys. Lett. A., math.QA/9806065.

[12] Avan J., Frappat L., Rossi M., and Sorba P.: submitted to Phys. Lett. A., math.QA/9807048. 